\title{
mTOR is involved in stroke-induced seizures and the anti-seizure effect of mild hypothermia
}

\author{
GUO-SHUAI YANG ${ }^{*}$, XIAO-YAN ZHOU* ${ }^{*}$ XUE-FANG AN, XUAN-JUN LIU, YAN-JUN ZHANG and DAN YU \\ Department of Neurology, Affiliated Haikou Hospital, Xiangya School of Medicine, \\ Central South University, Haikou, Hainan 570208, P.R. China
}

Received August 7, 2017; Accepted October 5, 2017

DOI: $10.3892 / \mathrm{mmr} .2018 .8629$

\begin{abstract}
Stroke is considered an underlying etiology of the development of seizures. Stroke leads to glucose and oxygen deficiency in neurons, resulting in brain dysfunction and injury. Mild hypothermia is a therapeutic strategy to inhibit stroke-induced seizures, which may be associated with the regulation of energy metabolism of the brain. Mammalian target of rapamycin (mTOR) signaling and solute carrier family 2, facilitated glucose transporter member (GLUT)-1 are critical for energy metabolism. Furthermore, mTOR overactivation and GLUT-1 deficiency are associated with genetically acquired seizures. It has been hypothesized that mTOR and GLUT-1 may additionally be involved in seizures elicited by stroke. The present study established global cerebral ischemia (GCI) models of rats. Convulsive seizure behaviors frequently occurred during the first and the second days following GCI, which were accompanied with seizure discharge reflected in the EEG monitor. Expression of phosphor (p)-mTOR and GLUT-1 were upregulated in the cerebral cortex and hippocampus, as evidenced by immunohistochemistry and western blot analyses. Mild hypothermia and/or rapamycin (mTOR inhibitor) treatments reduced the number of epileptic attacks, seizure severity scores and seizure discharges, thereby alleviating seizures induced by GCI. Mild hypothermia and/or rapamycin treatments reduced phosphorylation levels of mTOR and the downstream effecter p70S6 in neurons, and the amount of GLUT-1 in the cytomembrane of neurons. The present study revealed that mTOR is involved in stroke-induced seizures and the anti-seizure effect of mild hypothermia. The role of GLUT-1 in stroke-elicited seizures
\end{abstract}

Correspondence to: Professor Guo-Shuai Yang, Department of Neurology, Affiliated Haikou Hospital, Xiangya School of Medicine, Central South University, Haikou, Hainan 570208, P.R. China

E-mail: ygs_hk@163.com

"Contributed equally

Key words: mammalian target of rapamycin, stroke, seizures, mild hypothermia, solute carrier family 2 facilitated glucose transporter member 1 appears to be different from the role in seizures induced by other reasons. Further studies are necessary in order to elucidate the exact function of GLUT-1 in stroke-elicited seizures.

\section{Introduction}

Stroke has previously been demonstrated to be one of the most important underlying etiologies of the development of seizures (1). The brain comprises only $2 \%$ of human body mass, however consumes $25 \%$ of the body's total glucose and $20 \%$ of oxygen (2). Stroke results in glucose and oxygen deficiency for brain cells, leading to brain dysfunction and injury $(1,2)$. The specific mechanisms by which stroke results in the occurrence and development of seizures remain to be fully elucidated, however abnormal neuronal hyperexcitability is commonly observed following stroke (3). A total of $50-70 \%$ of seizures are observed in children $<1$ year of age and adults $>60$ years (4). Clinical data suggest that seizures are a commonly observed complication following perinatal and childhood stroke $(5,6)$. In addition, stroke, whether ischemic or hemorrhagic, is an independent risk factor for seizures in the elderly population (7). Seizures occurring in this age group are associated with an increased risk of mortality during the first year following the onset of the seizure (4), therefore a better understanding regarding the pathological mechanisms and further improvements in treatment modalities are of primary concern.

Mild hypothermia of the brain or the whole body is a therapeutic strategy to alleviate stroke burden by enhancing tolerance of neurons to glucose and oxygen deficiency. Mild hypothermia of the whole body (rectal or esophageal temperature $33-34^{\circ} \mathrm{C}$ ), which starts within $6 \mathrm{~h}$ following birth and protracts for 48-72 h, significantly reduces the incidences of death or disability at 18 months, with improved neurological outcome in survivors $(8,9)$. Ongoing studies reveal that hypothermia is able to inhibit the occurrence and frequency of seizures induced by stroke. Seizures following human-perinatal stroke are effectively avoided when neonates are born in a cooling environment (10). Evidence from animal data indicates that hypothermia is significantly associated with a lower frequency of seizures in acute stroke (11). The mechanism by which hypothermia conveys anti-seizure effects remains to be fully elucidated, although it is known that hypothermia attenuates excitotoxicity, specifically the 
glutamate- and dopamine-associated cerebral cytotoxicity of global ischemia (12).

The mammalian target of rapamycin (mTOR) is a serine/threonine kinase involved in the highly conserved phosphatidylinositol-3-kinase (PI3K)-AKT serine/threonine kinase (AKT) signaling pathway (13). mTOR has been reported to regulate multiple physiological processes of neurons and glia, including their growth and survival, metabolism and autophagy, in addition to structure and cell-cell interactions. However, hyperactivation of mTOR signaling has been associated with certain types of seizures resulting from genetic mutation and brain injury (13). Excessive activation of mTOR is usually the consequence of mutations in negative regulators of mTOR, including phosphatase and tensin homolog and tuberous sclerosis complexes 1 and 2 (TSC1 and TSC2), in a class of human neurological diseases collectively referred to as 'TORopathies' (13). mTOR overactivation results in cortical malformations and epileptic phenotypes. Inhibition of mTOR by rapamycin, everolimus, tamoxifen or SB-399885 consistently attenuates seizures in various genetic and acquired seizure animal models, which suggests a close association between mTOR and these types of seizures $(1,14,15)$. However, it is currently unclear whether mTOR is implicated in the pathogenesis of stroke-induced seizures.

Glucose is the primary energy source for the brain, however, due to its hydrophilic property, it needs to be transported across the cell membrane via glucose transporters (GLUTs), prior to being utilized by neurons and glial cells (16). Solute carrier family 2 , facilitated glucose transporter member (GLUT)-1 is the predominant transporter responsible for glucose transport in the brain (16). GLUT-1 deficiency (G1D) syndrome, which is induced by GLUT-1 mutation, is associated with neurological dysfunction and paradoxically excessive neuronal activation (presented as spike-wave seizures) (17). Ullner et al (18) constructed a haploinsufficient GLUT-1 mouse model (Glut-1 ${ }^{+/}$), in which epileptiform discharges were observed on the electroencephalogram (EEG). These data suggest that GLUT-1 deficiency is an important contributor to seizures. Expression and intracellular translocation of GLUT-1 are reported to be regulated by the mTOR signaling pathway (19). Various types of cancers result in overactivation of PI3K/AKT/mTOR cascades that are associated with upregulated GLUT-1 in cells and the increased glucose uptake (20). mTOR activation induces upregulation of hypoxia inducible factor and vascular endothelial growth factor and acceleration of protein synthesis, which may increase GLUT-1 abundance in cells (21). However, it has additionally been demonstrated that mTOR activation resulting from loss of TSC2 function $\left(\mathrm{Tsc}^{-/}\right.$) diminishes glucose uptake by the embryonic fibroblasts via inhibition of GLUT translocation from the cytoplasm to plasma membrane (22). Therefore, mTOR promotes or inhibits glucose uptake by cells through different mechanisms regulating GLUTs. mTOR regulation of GLUTs and the glucose uptake is dependent on the cell type and surrounding environments.

The present study aimed to investigate whether stroke-induced seizures are associated with hyperactivation of mTOR in neurons. The study also aimed to determine whether the protective effect of hypothermia against seizures is associated with mTOR inhibition. Finally, the mechanism underlying how mTOR regulates GLUT-1 in stroke-induced seizures and the hypothermic condition was investigated. Overall, the study aimed to facilitate further understanding of the pathogenesis of stroke-induced seizures and the improvement of hypothermic therapy for the future.

\section{Materials and methods}

Animals. A total of 105 Sprague-Dawley male rats (8-10 weeks, $\sim 280 \mathrm{~g}$; Central South University, Changsha, China) were separately housed in four vivariums that were maintained at a fixed temperature $\left(22-23^{\circ} \mathrm{C}\right)$ and moisture $(70 \%)$, with a 12 -h light on/off cycle. Food and water were provided ad libitum. All procedures were approved by the Animal Care and Use Committee of the Central South University and adhered to National Institutes of Health Guidelines for the Care and Use of Animals (23). In line with the guidelines, animals with severe seizures were treated with clinically appropriate anticonvulsants, however these animals were excluded from the experiments.

Global cerebral ischemia (GCI). GCI was performed using an ' $L$ ' shape stick, the method of which was described in a previous study (24). Briefly, animals were anesthetized with $2 \%$ isoflurane and subsequently placed in a supine position with the four extremities fastened to the table. The ' $L$ ' shape stick was inserted into the mediastinum at the level of the second intercostal segment and the distal end of the stick was twisted $45^{\circ}$ for positioning under the bundle of primary cardiac blood vessels. To interrupt blood flow to the brain, the stick was lifted up with finger pressure from outside of the chest. The compression for 2-3 min resulted in cardiac arrest. Rats were immediately treated with a rodent respirator to help recover heart beat and blood supply to the brain. Rats $(n=9)$ in the sham group (Nor) were subjected to the same procedure without compression of the carotid arteries and served as the control.

Experimental design. Following establishment of GCI, rats with convulsive seizures were randomly selected and humanely euthanized at indicated time-points $(24,48 \mathrm{~h}$, and 7 days, $n=3$ each time). Protein levels of mTOR and GLUT-1 in specific regions of the brain were tested using a western blot analysis. In addition, following GCI, a group of rats were randomly divided into 4 groups: i) GCI group (no further treatment, $n=18)$; ii) mild hypothermia group $(n=24)$; iii) rapamycin group ( $\mathrm{n}=24)$; and iv) mild hypothermia+rapamycin group $(n=30)$. Rats in the last three groups were subjected to treatments with mild hypothermia and rapamycin injection, alone or in combination.

Mild hypothermia. Active whole-body cooling was performed via a blanket cooling device (Cincinnati Subzero Blanketrol III, Cincinnati, OH, USA) immediately following the GCI. Core temperatures of rats, as measured by a rectal probe, were maintained at $33.5^{\circ} \mathrm{C}$ for $30 \mathrm{~min}$. At the end of the cooling period, the animal was transferred to a heating pad and allowed to warm up to normal body temperature. Following this, the animal was released into the normal housing cage. 
Rapamycin injection. Rapamycin injections were administered following the method described by Butler et al (15). Rapamycin (LC Laboratories, Woburn, MA, USA) was initially dissolved in $100 \%$ ethanol $(20 \mathrm{mg} / \mathrm{ml})$ and then diluted in a vehicle solution containing 5\% Tween-80, 5\% PEG400, and 4\% ethanol (Thermo Fisher Scientific, Inc. Waltham, MA, USA) dissolved in distilled, deionized water. Rapamycin (3 $\mathrm{mg} / \mathrm{kg}$ ) or vehicle was injected intraperitoneally when the mice regained consciousness following the GCI injury (20-30 min) and the treatment was continued once daily until rats were sacrificed.

Seizure severity scores and EEG recordings. To detect seizures following GCI, animals were placed under continuous visual surveillance with concurrent EEG recordings. The appearance of seizures was exemplified by rapid running, jumping, barrel rolling ( $\geq 3$ turns), falling (loss of righting reflex) with tonic limb flexion, and repetitive tail erection. The seizure severity was evaluated with a scoring method (25): $0=$ normal behavior; 1 = immobility; 2 = spasm, tremble, or twitch; 3 = tail extension; 4 = forelimb clonus; 5 = generalized clonic activity; $6=$ jumping or running seizures; 7 = full tonic extension and $8=$ death. The seizure severity scores were given by a neurologist blinded to treatments and to time post-injury.

EEG recordings were performed in free-moving animals using an amplitude-integrated EEG monitor as previously described (26). A rat restrainer was used when rats exhibited vigorous convulsive behavior, including jumping and rapid running. EEG recordings were performed using a dual-channel AC microelectrode amplifier connected to a custom-built digital video-EEG monitoring system (model 1,800; AM Systems, Carlsborg, WA, USA). Electrodes were implanted bilaterally into the hippocampal CA1 (bregma-2.3 mm, lateral $2.0 \mathrm{~mm}$ and depth $2.0 \mathrm{~mm}$ ) and parietal cortex (bregma- $0.6 \mathrm{~mm}$, lateral $1.5 \mathrm{~mm}$ and depth $1 \mathrm{~mm})$. Signals were collected in a frequency bandwidth of 0.1-1,000 Hz, amplified 1,000 times and then digitized at $\geq 5 \mathrm{KHz}$ (Digidata 1,300; Molecular Devices, LLC, Sunnyvale, CA, USA). Data were analyzed using pClamp software, version 10 (Molecular Devices, LLC).

Immunohistochemistry (IHC). Following sacrifice of rats, the cerebral cortex and hippocampus were separated from the brain, fixed in $10 \%$ formalin with $20 \%$ sucrose at room temperature for $12 \mathrm{~h}$ and finally embedded in paraffin. The sections $(5 \mu \mathrm{m})$ were blocked with PBS containing $0.3 \%$ Triton X-100/5\% bovine serum albumin (w/v, Beijing Solarbio Science and Technology, Co., Ltd., Beijing, China) for $1 \mathrm{~h}$ at room temperature, prior to incubation with primary antibodies specific for phospho(p)-mTOR (Ser2448; 1:1,000 dilution; cat. no. 2971; Cell Signaling Technology, Inc., Danvers, MA, USA) and GLUT-1 (1:1,000 dilution, ab32551; Abcam Cambridge, $\mathrm{UK}$ ) for $2 \mathrm{~h}$ at room temperature. The sections were then incubated with horseradish peroxidase (HRP)-labeled anti-IgG secondary antibody (1:2,000 dilution; PA128664, Invitrogen; Thermo Fisher Scientific, Inc.) for $30 \mathrm{~min}$ at room temperature, and treated with Thermo Scientific ${ }^{\mathrm{TM}}$; Pierce $^{\mathrm{TM}}$ ECL solution (Pierce; Thermo Fisher Scientific, Inc.). All the sections were counterstained with haematoxylin for $30 \mathrm{~min}$ at room temperature and analyzed using a microscope (Axio Imager 2; Carl Zeiss AG, Oberkochen, German).
Western blot analysis. Cell membrane proteins of the cerebral cortex and hippocampus tissues were extracted using a membrane protein extraction kit (Biovision, Inc., Milpitas, CA, USA). The membrane proteins were subsequently used for western blot analysis of GLUT-1 and $\mathrm{Na}^{+} / \mathrm{K}^{+}$-ATPase. Detection of proteins in the cells of the cerebral cortex and hippocampus tissues occurred via homogenizing the tissues directly using a lysis buffer (10 mM HEPES pH 7.9, $1.5 \mathrm{mM}$ $\mathrm{MgCl}_{2}, 10 \mathrm{mM} \mathrm{KCl}, 12 \%$ glycerol, $0.1 \mathrm{mM}$ EGTA, $0.5 \mathrm{mM}$ DTT, and $0.5 \mathrm{mM}$ spermidine) with the addition of a protease inhibitor cocktail (Sigma-Aldrich; Merck KGaA, Darmstadt, Germany). Proteins were quantified via a Bicinchoninic Acid assay (Pierce; Thermo Fisher Scientific, Inc.). Proteins $(20 \mu \mathrm{g})$ were separated by $12 \%$ SDS-PAGE in Tris buffer, prior to being transferred to nitrocellulose membranes. Following blocking with $5 \%$ non-fat milk overnight at $4^{\circ} \mathrm{C}$, membranes were incubated with an anti-GLUT-1 antibody (cat. no. ab32551, 1:1,000 dilution; Abcam), anti-p-AMP-activated protein kinase (AMPK) antibody (Thr172, cat. no. ab133448, 1:1,000 dilution; Abcam), anti-mTOR antibody (cat. no. 2972; 1:1,000 dilution), and anti-p-mTOR antibody (Ser2448, cat. no. 2971; 1:1,000 dilution) (both from Cell Signaling Technology, Inc.), anti-p-p70S6 kinase (S6K; Thr229) antibody (cat. no. GTX25231, 1:1,000 dilution; GeneTex, Inc., Irvine, CA, USA), anti-p-AKT (T308) antibody (cat. no. ab38449, 1:1,000 dilution; Abcam), anti-p-p70S6 kinase antibody (cat. no. ab65753, 1:500 dilution; Abcam), or anti-GAPDH antibody (cat. no. SC-365062, 1:800 dilution; Santa Cruz Biotechnology, Inc., Dallas, TX USA) overnight at $4^{\circ} \mathrm{C}$. Then, membranes were incubated with a HRP-conjugated secondary antibody that targeted mouse IgG (cat. no. ab97040; Abcam), rabbit IgG (cat. no. A0545), or goat IgG (cat. no. A5420) (both from Sigma-Aldrich; Merck KGaA) at a 1:2,000 dilution for $2 \mathrm{~h}$ at room temperature. Reactive proteins were detected using Enhanced Chemiluminescent and SuperSignal ${ }^{\mathrm{TM}}$ Chemiluminescent substrates (Pierce; Thermo Fisher Scientific, Inc.), and quantified using Optiquant 3.0 software (PerkinElmer, Inc., Waltham, MA, USA).

Statistical analysis. Statistical tests were conducted using SPSS software, version 12.0 (SPSS, Inc., Chicago, IL, USA). Data are presented as the mean \pm standard deviation. A Student's t-test was used for two group comparisons. For multiple group comparisons, a one-way analysis of variance followed by Dunn's test was used. $\mathrm{P}<0.05$ was considered to indicate a statistically significant difference.

\section{Results}

GCI provokes seizures in rats. In the present study, seizures were observed during the first $24 \mathrm{~h}$ following GCI, however not in the sham surgery group. The number of seizure attacks was increased during the second $24 \mathrm{~h}$ following GCI $(\mathrm{P}<0.05$; Fig. 1A), compared with the first $24 \mathrm{~h}$ following GCI. The number of seizure attacks from day 6 to 7 following GCI was reduced compared with the first and the second $24 \mathrm{~h}$ following GCI. Rats gained relative high seizure severity scores during the first and the second $24 \mathrm{~h}$ following GCI, however the scores were decreased from day 6 to 7 following GCI $(\mathrm{P}<0.05$; Fig. 1B). EEG discharges of seizures were defined as repetitive 
A

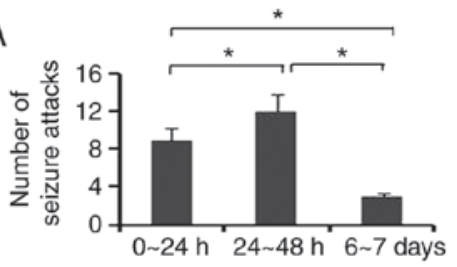

$\mathrm{B}$

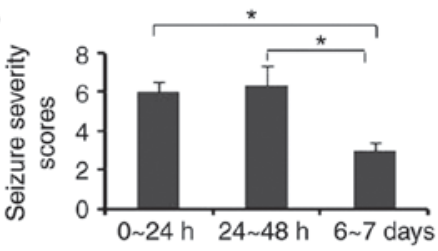

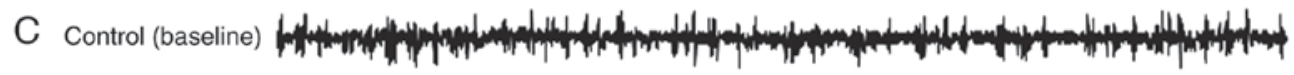
$24 \mathrm{~h}$ after GCl 48 a after GCl

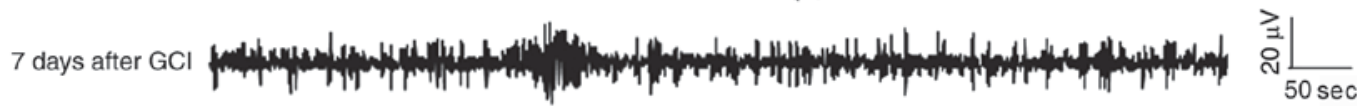

Figure 1. GCI provokes seizures in rats. (A) The number of seizure attacks, (B) seizure severity scores and (C) electroencephalo-graph recordings were used to evaluate seizures at different time periods following GCI. "P<0.05 as indicated. GCI, global cerebral ischemia.

(C)

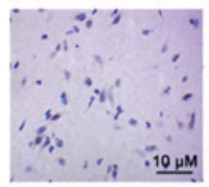

p-mTOR

(H)

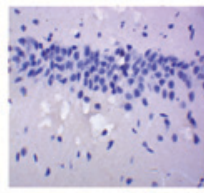

(C)

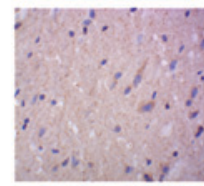

GLUT-1

(H)

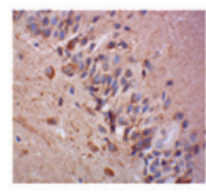

Nor
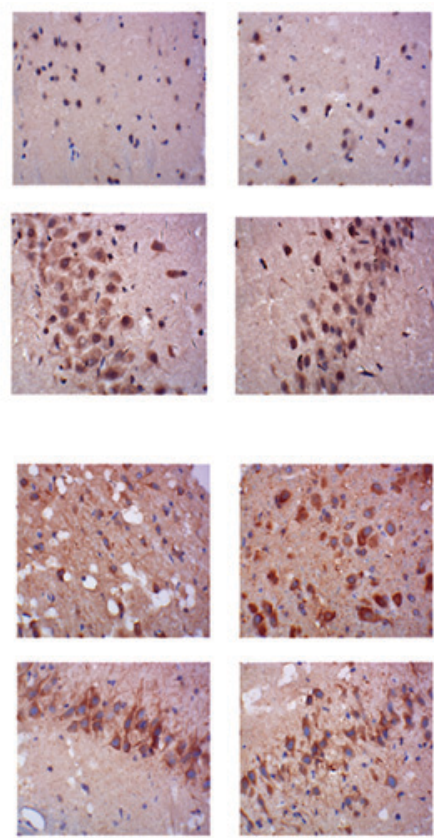

$24 \mathrm{~h}$
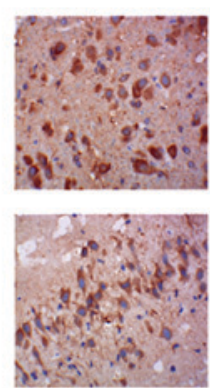

$48 \mathrm{~h}$
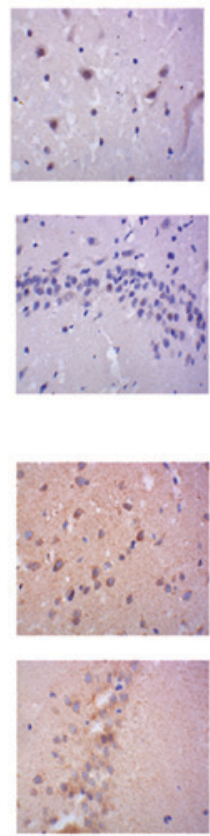

7 days

(X400)

Figure 2. p-mTOR and GLUT-1 expression in neurons following stroke. Immunohistochemistry was conducted to detect expression of p-mTOR and GLUT-1 in neurons in cerebral cortex and hippocampus of rats at three time-points, $24,48 \mathrm{~h}$ and 7 day post-surgery. Magnification, $\mathrm{x} 400$. C, cerebral cortex tissues; H, hippocampus; Nor, normal group; GCI, global cerebral ischemia; p, phosphorylated; mTOR, mammalian target of rapamycin; GLUT-1, solute carrier family 2 , facilitated glucose transporter member 1 .

single-spike or poly-spike waveforms lasting $\geq 25 \mathrm{sec}$ in duration with amplitudes $\geq x 2$ that of the background signals. As presented in Fig. 1C, typical EEG discharges of seizures were observed following GCI, with the longest duration and highest amplitudes presented during $24-48 \mathrm{~h}$ following GCI. The duration and amplitudes of EEG discharges of seizures were decreased 7 days following GCI, compared with those during 24-48 $\mathrm{h}$ following GCI.

Upregulation of mTOR and GLUT-1 in neurons following stroke. Immunohistochemistry was conducted to detect expression of p-mTOR and GLUT-1 in neurons from the 
cerebral cortex and hippocampus of rats at three time-points, $24,48 \mathrm{~h}$ and 7 day post-surgery. Samples from rats that were not subjected to GCI were treated as control (Nor) group. Compared with Nor group, p-mTOR staining was notably increased in the cytoplasm and nucleus of neurons in the cerebral cortex and hippocampus at $24 \mathrm{~h}$ and $48 \mathrm{~h}$ following GCI (Fig. 2). A total of 7 day following GCI, a small fraction of neurons in the cerebral cortex and hippocampus still revealed moderate p-mTOR staining. GLUT-1 staining presented outside of neuron nucleus. GLUT-1 staining in neurons in the cerebral cortex was increased following GCI, with a peak level at $48 \mathrm{~h}$. On day 7, 70\% neurons demonstrated relatively deep GLUT-1 staining. GLUT-1 staining in neurons in the hippocampus was increased at 24 and $48 \mathrm{~h}$, however not on day 7 , following GCI.

Furthermore, mTOR, p-mTOR and GLUT-1 protein levels in the cerebral cortex and hippocampus detected via western blotting were quantified. As presented in Fig. 3, mTOR and p-mTOR protein levels were increased at 24 and 48 h post-surgery, compared with Nor group $(\mathrm{P}<0.05)$. mTOR protein level was still increased on day 7 following GCI compared with Nor group. GLUT-1 functions as a glucose transporter following its transfer to the cell membrane from the cytoplasm. Therefore, the present study examined the abundance of GLUT-1 only in neuronal membranes, to evaluate the ability to transport glucose. The GLUT-1 protein level in the cell membrane was increased at 24 and $48 \mathrm{~h}$ following GCI $(\mathrm{P}<0.05)$, compared with Nor group. There was no significant difference between control value and the GLUT-1 protein level 7 day following GCI.

Mild hypothermia and rapamycin treatments alleviate seizures induced by GCI. The number of epileptic attacks and seizure severity scores during the second $24 \mathrm{~h}$ following GCI were decreased by mild hypothermia and rapamycin treatments, alone or in combination $(\mathrm{P}<0.05$; Fig. $4 \mathrm{~A}$ and $\mathrm{B})$. In addition, EEG recordings revealed that mild hypothermia and rapamycin treatments suppressed the burst of the epileptic discharge, and resulted in a reduction in the spike frequency and amplitude (Fig. 4C).

Hypothermia and rapamycin treatments inhibit upregulation of p-mTOR and GLUT-1 following stroke. Immunohistochemistry demonstrated that immediate hypothermia following GCI prevented the increase in p-mTOR staining in neurons from the cerebral cortex and hippocampus, at $48 \mathrm{~h}$ following GCI (Fig. 5). The injection of rapamycin more effectively inhibited the increase in the staining of p-mTOR than hypothermia; the effects of hypothermia against seizures may be associated with other mechanisms in addition to mTOR inhibition. Combination of the hypothermia and rapamycin injection demonstrated a synergistic effect on the inhibition. The hypothermic strategy also hindered the increase in GLUT-1 staining in neurons in the cerebral cortex and hippocampus. Furthermore, the mTOR inhibitor rapamycin exerted a strong suppressive effect on the GLUT-1 staining, particularly in neurons from the hippocampus. The combination of hypothermia and rapamycin injection demonstrated a synergistic effect on the inhibition, particularly in neurons from the cerebral cortex.
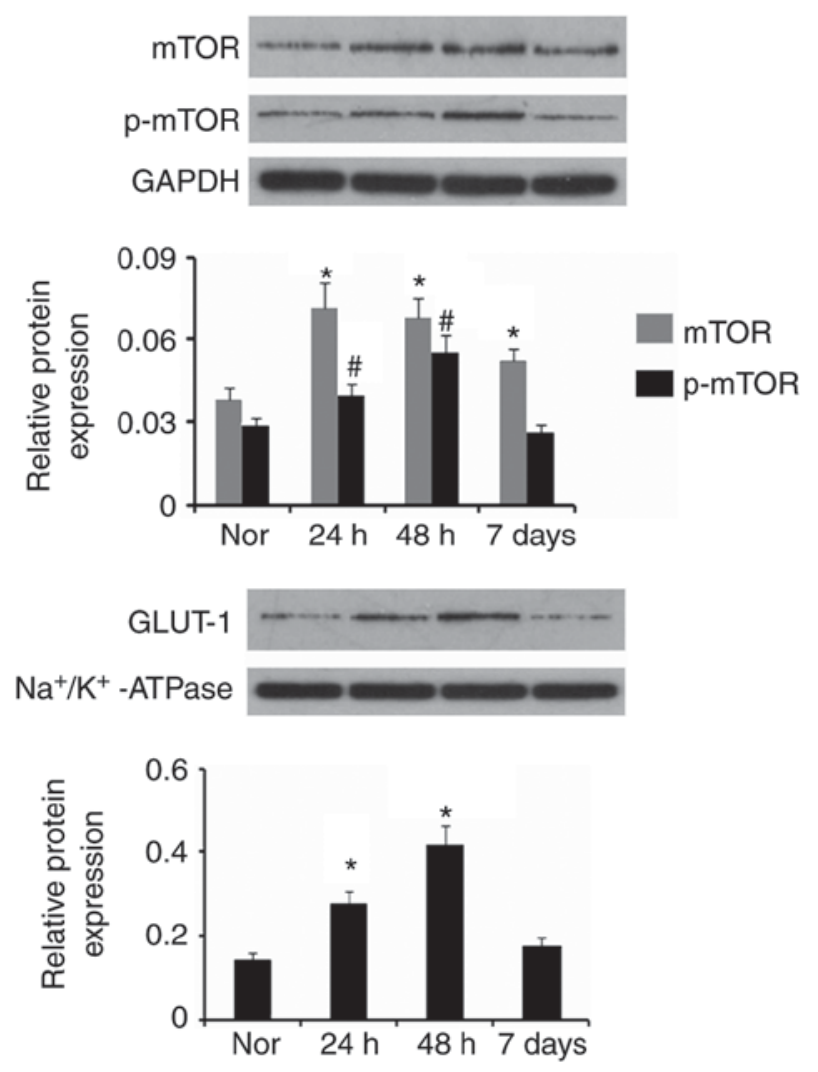

Figure 3. mTOR, p-mTOR and GLUT-1 expression in neurons following stroke. Western blotting was conducted to detect the expression levels of mTOR, p-mTOR and GLUT-1 in neurons and the cell membrane, respectively, in the cerebral cortex and hippocampus of rats at three time-points, $24,48 \mathrm{~h}$ and 7 day post-surgery. ${ }^{*} \mathrm{P}<0.05$ and ${ }^{~} \mathrm{P}<0.05$ vs. control (Nor). Nor, normal group; $\mathrm{p}$, phosphorylated; mTOR, mammalian target of rapamycin; GLUT-1, solute carrier family 2, facilitated glucose transporter member 1 .

Similar to the immunohistochemistry outcome, western blotting demonstrated that hypothermia prevented the increase in mTOR protein levels in the cerebral cortex and hippocampus from GCI, and even reversed the $\mathrm{p}-\mathrm{mTOR}$ protein level $(\mathrm{P}<0.05$ vs. Nor group; Fig. 6A). Rapamycin abolished the increase in p-mTOR protein level by GCI, however only moderately inhibited the increase in mTOR. The combination of hypothermia and rapamycin injection decreased protein levels of mTOR and p-mTOR, compared with Nor group $(\mathrm{P}<0.05)$. Protein levels of p-p70S6 and p-AKT, which are the downstream effecter and upstream regulator, respectively, of mTOR were additionally detected. p-p70S6 protein level in cerebral cortex and hippocampus was increased by GCI. Hypothermia following GCI decreased the p-p70S6 protein level compared with Nor group $(\mathrm{P}<0.05)$. Treatment with rapamycin abolished increase in p-p70S6 protein level by GCI. Co-treatment of hypothermia and rapamycin following GCI significantly decreased the p-p70S6 protein level. GCI upregulated p-AKT protein level in cerebral cortex and hippocampus. Although hypothermia to a certain extent decreased the p-AKT protein level, it was still significantly increased compared with Nor group $(\mathrm{P}<0.05)$. Treatment with rapamycin further increased the $\mathrm{p}$-AKT protein level $(\mathrm{P}<0.05)$. Combination of hypothermia and rapamycin treatments exerted a modest effect on the increase in p-AKT protein level. p-AMPK protein level demonstrated no significant alterations between groups. 
A

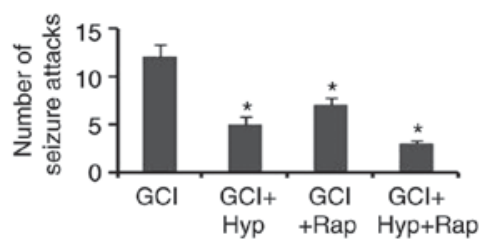

B

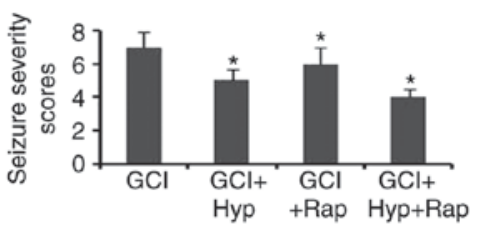

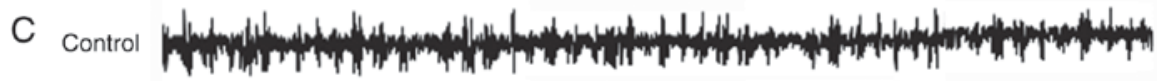

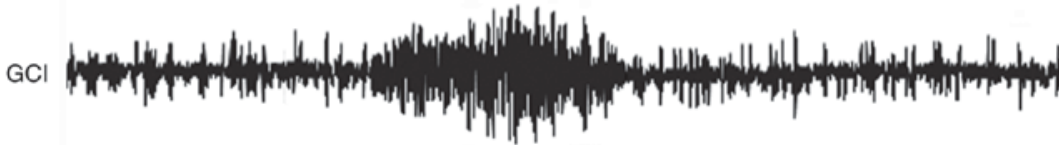

$\mathrm{GCl}+\mathrm{Hyp}$

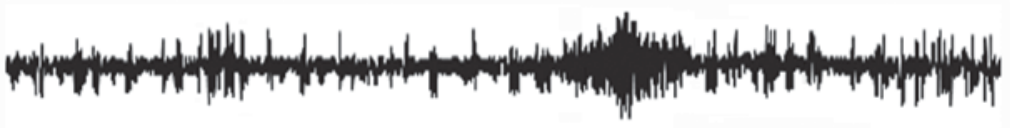

$\mathrm{GCl}+\mathrm{Rap}$

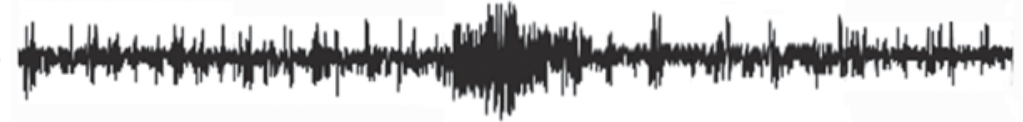

GCl+Hyp+Rap

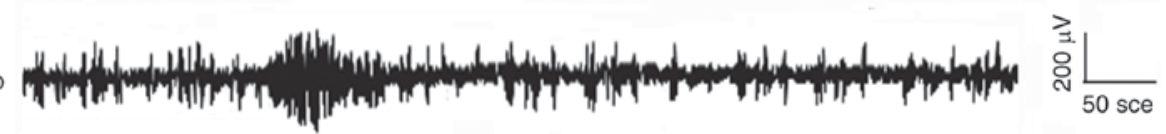

Figure 4. Mild hypothermia and rapamycin treatments alleviate seizures induced by GCI. (A) The number of seizure attacks, (B) seizure severity scores and (C) electroencephalo-graph recordings were used to evaluate the intensity of seizures following different treatments. "P<0.05 vs. GCI group. GCI, global cerebral ischemia; Hyp, mild hypothermia treatment; Rap, rapamycin injection.

(C)

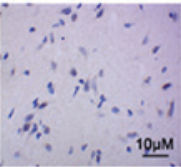

p-mTOR

(H)

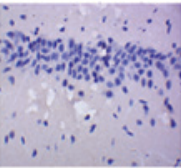

(C)

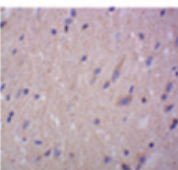

GLUT-1

(H)

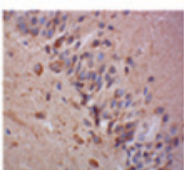

Nor
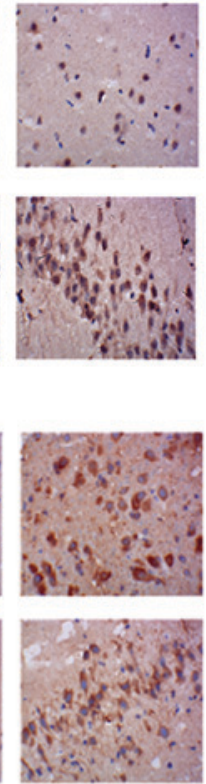

$\mathrm{GCl}$
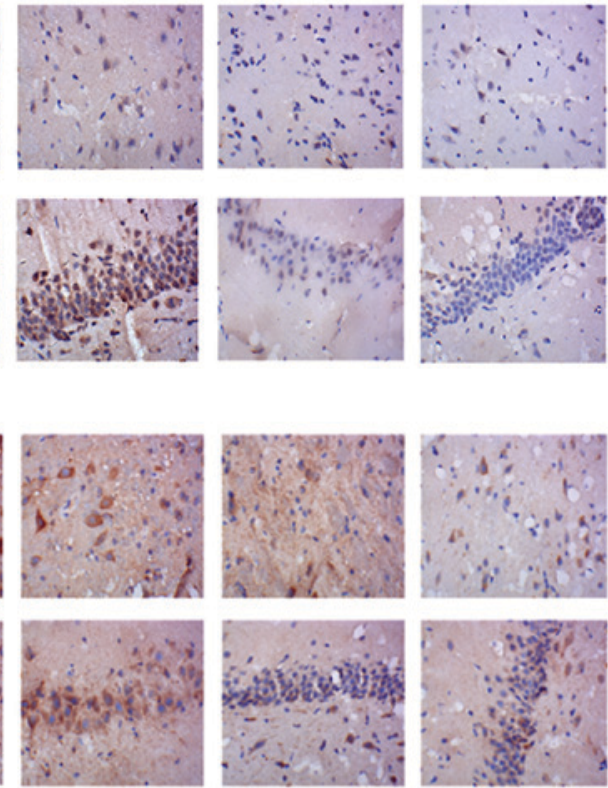

$\mathrm{GCl}+\mathrm{Hyp}$

(X400)

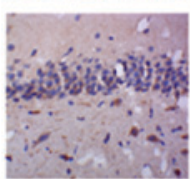

$\mathrm{GCl}+\mathrm{Rap}$

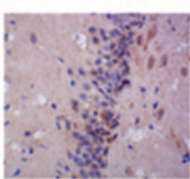

$\mathrm{GCl}+\mathrm{Rap}+$

Hyp

Figure 5. p-mTOR and GLUT-1 expression following hypothermia and rapamycin treatments. Following GCI, rats were subjected to active whole-body cooling and/or rapamycin injection protocols. Immunohistochemistry was conducted to detect expression of p-mTOR and GLUT-1 in neurons in the cerebral cortex and hippocampus of rats at $48 \mathrm{~h}$ following GCI. C, cerebral cortex tissues; H, hippocampus; Nor, normal group; GCI, global cerebral ischemia; Hyp, mild hypothermia treatment; Rap, rapamycin injection; p, phosphorylated; mTOR, mammalian target of rapamycin; GLUT-1, solute carrier family 2, facilitated glucose transporter member 1 .

GLUT-1 protein level was significantly increased in cell membrane of neurons in cerebral cortex and hippocampus following GCI $(\mathrm{P}<0.01$; Fig. $6 \mathrm{~B})$, however this increase was abolished by post-treatment with hypothermia. Post-treatment with rapamycin additionally inhibited the increase in GLUT-1 protein level, however it was still increased compared with Nor 
A

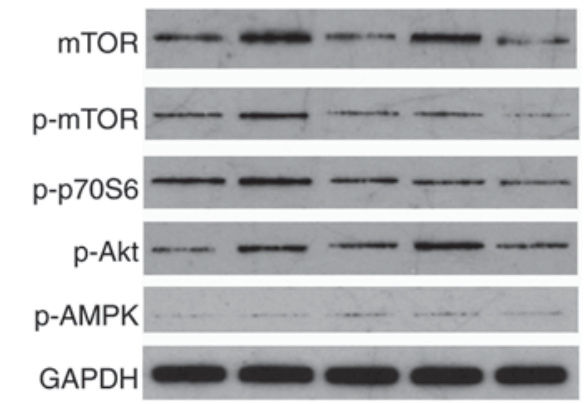

B

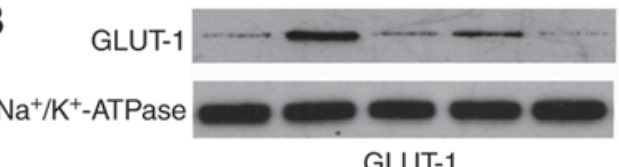

GLUT-1

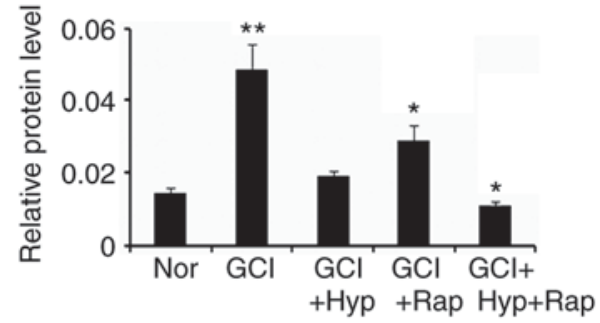

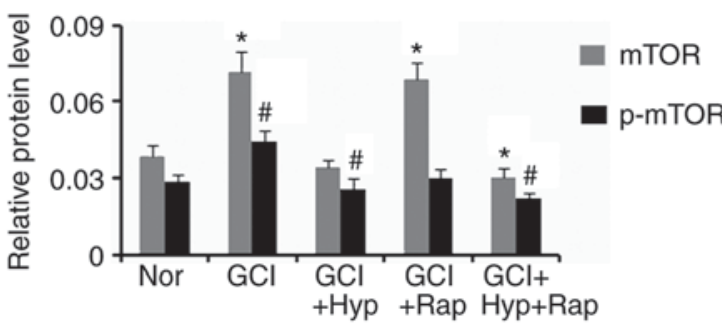
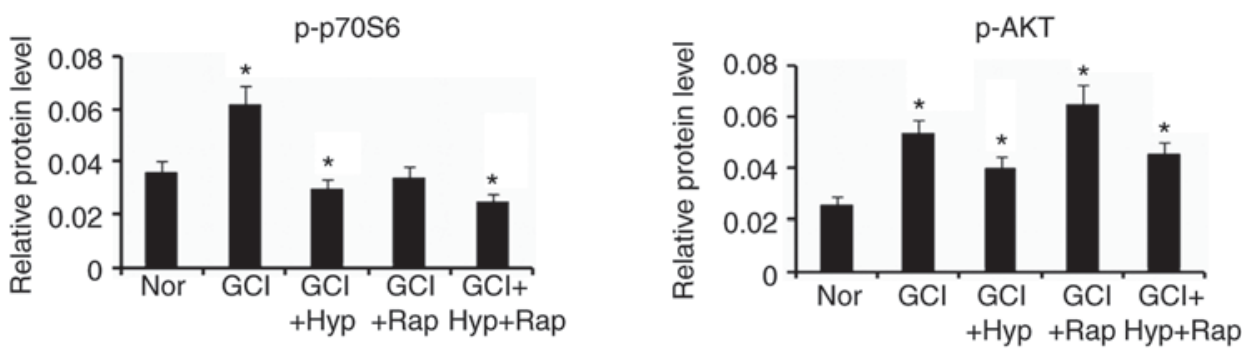

Figure 6. Protein expression levels following hypothermia and rapamycin treatments. Following GCI, rats were subjected to active whole-body cooling and/or rapamycin injection protocols. (A) Expression levels of proteins were detected in neurons in cerebral cortex and hippocampus of rats, (B) except for GLUT-1 protein levels that were detected in the cell membrane of neurons, at $48 \mathrm{~h}$ following GCI by western blotting. Nor, normal group; GCI, global cerebral ischemia; Hyp, mild hypothermia treatment; Rap, rapamycin injection; p, phosphorylated; mTOR, mammalian target of rapamycin; GLUT1, solute carrier family 2 , facilitated glucose transporter member 1 ; Akt, AKT serine/threonine kinase; AMPK, AMP-activated protein kinase. ${ }^{*} \mathrm{P}<0.05$, ${ }^{* *} \mathrm{P}<0.01$ and ${ }^{\#} \mathrm{P}<0.05$ vs. control (Nor).

group $(\mathrm{P}<0.05)$. The combination of hypothermia and rapamycin treatments decreased the GLUT-1 protein level $(\mathrm{P}<0.05)$.

\section{Discussion}

Stroke has been identified as an important causative factor for seizures, however the underlying mechanisms have not been fully elucidated. It is known that brain cells are very vulnerable to energy deficiency in stroke, therefore it is reasonable to hypothesize that signaling molecules that participate in regulation of energy metabolism may be involved in the pathological mechanism of post-stroke seizures. mTOR is an intriguing candidate due to its critical role in energy and protein metabolism. In addition to this, loss of function of inhibitors upstream of the mTOR pathway have previously been demonstrated to be associated with genetically acquired development of seizures (13-15). The present study aimed to investigate the involvement of the mTOR pathway in stroke-associated seizures. A rat stroke model was first established via the arrest of primary vessels that supply blood to the brain. Convulsive seizure behaviors frequently occurred during the first and the second $24 \mathrm{~h}$ following GCI, which were accompanied with seizure discharge reflected in the EEG monitor. This evidence indicated that stroke resulted in seizure occurrences. mTOR was upregulated in neurons in the cerebral cortex and hippocampus in response to GCI. Parenteral administration of the mTOR inhibitor rapamycin repressed phosphorylation levels of mTOR and its downstream target S6 protein, and inhibited post-ischemic seizure-associated characteristics, which suggested that mTOR was involved in stroke-associated seizures.

The outcome of the increased mTOR activity following stroke did not conform to anticipated results, as stroke has previously been demonstrated to activate AMPK through decreasing ATP/AMP ratio in neurons (27). Activated AMPK is associated with diminished mTOR activity due to the stimulatory effect of AMPK on TSC1/2. However, the results of the western blotting assay demonstrated that the phosphorylation level of AMPK at Thr172 in the cerebral cortex and hippocampus tissues did not increase at $48 \mathrm{~h}$ following stroke. Fu et al (28) using western blotting and immunohistochemistry suggest that phosphorylated AMPK significantly increases at 3 and $6 \mathrm{~h}$ following focal ischemia in the cerebral cortex of mice, however decreases at 12 and $24 \mathrm{~h}$. This indicates that increased AMPK activity is observed a short time period following stroke. PI3K-AKT represents a key signaling pathway positively regulating mTOR activity. It was observed that AKT phosphorylation was significantly elevated at $48 \mathrm{~h}$ following stroke, which indicated that the increased mTOR activity was associated with the stimulation of AKT. 
Mild hypothermia of the brain following stroke has been verified to effectively attenuate the severity and frequency of seizures (29). It is generally believed that the inhibitory effect of hypothermia on seizures is associated with the reduction of energy metabolism which enhances the tolerance of neurons under the status of glucose and oxygen deficiency and prevents disorders of transmission of neural signals in stroke conditions (29-31). According to previous studies, cerebral metabolic rate is reduced by $6-7 \%$ per degree Celsius reduction. Dropping temperatures to $20^{\circ} \mathrm{C}$ leads to cerebral metabolic rate of one fifth of that at normothermic values (29-31). As a consequence, the release of excitatory neurotransmitters is slowed and the function of ion pumps in the neuron membrane is altered, which are proposed to contribute to seizure suppression (30-32). However, the molecular basis underlying how hypothermia alleviates seizures has rarely been investigated. In the present study, hypothermia prevented mTOR activation with functional consequence equivalent to treatment with rapamycin. Furthermore, hypothermia in combination with rapamycin treatment exhibited a synergistic effect on mTOR suppression, which resulted in a more efficient therapeutic effect on seizures compared with hypothermia and rapamycin treatments alone. Decreased mTOR activity from hypothermia may be involved in its protective effect against seizures post-stroke.

The critical role of GLUT-1 in delivering glucose to neurons is well established. GLUT-1 deficiency in brain cells is associated with a series of neurological diseases including seizures (19). The present study detected the abundance of GLUT-1 in neurons via immunohistochemistry and in the cell membrane via western blotting. Notably, GLUT-1 expression was upregulated in neurons at 24 and $48 \mathrm{~h}$ following stroke. This alteration may have been an adaptive response for neurons that underwent GCI. Inhibition of metabolism in the brain by hypothermia treatment did not result in a notable increase in GLUT1 expression following stroke. GLUT1 expression and translocation from cytoplasm to cell membrane are regulated by mTOR via its complicated biological functions (19). Treatment of the rats with mTOR inhibitor following stroke resulted in decreased GLUT1 abundance in the neurons and the cell membrane, suggesting that mTOR promoted GLUT1 amplification following stroke and facilitated glucose uptake by neurons. It has been hypothesized that blood supply to the brain was recovered 3 min following vascular compression, thus the situation of glucose and oxygen deficiencies in cerebral neurons at 24 and $48 \mathrm{~h}$ following stroke did not occur. However, damages induced by preceding stroke were not eliminated at these time-points, and GLUT1 was unable to repair the detriments just through replenishing energy for neurons. Therefore, GLUT1 upregulation triggered by mTOR would result in a weak effect on seizure suppression. Conversely, various biological functions of mTOR, may contribute to seizures $(14,15)$, thus inhibition of mTOR activity either by drug-like molecules or a physical approach (hypothermia) is desirable to attenuate seizures following stroke.

In conclusion, the present study revealed that the seizures induced by stroke were associated with mTOR activation, and hypothermia protected against the seizures via repressing mTOR. Although mTOR increased GLUT-1 abundance in cell membrane of neurons following stroke, GLUT-1 had a limited effect on seizures following stroke. Results obtained in the present study contribute to further understanding of pathogenesis of stroke-induced seizures and improvement of hypothermic therapy in the seizures.

\section{Acknowledgements}

Not applicable.

\section{Funding}

The present study was supported by the Key Program of Science and Technology Planning of Haikou (grant no. 2014-73) and Hainan Provincial Natural Science Foundation (grant no. 20158272).

\section{Availability of data and methods}

All datasets used in the current study are available upon request from the corresponding author

\section{Authors' contributions}

GY and XZ took the responsibility of the experimental design. GY, XA, XL, YZ and DY performed the study and wrote the manuscript. All authors have read and approved of the final manuscript.

\section{Ethics approval and consent to participate}

All procedures were approved by the Animal Care and Use Committee of the Central South University and adhered to National Institutes of Health Guidelines for the care and use of animals (23).

\section{Consent for publication}

Not applicable.

\section{Competing interests}

The authors declare that they have no competing interests.

\section{References}

1. Stefanidou M, Das RR, Beiser AS, Sundar B, Kelly-Hayes M, Kase CS, Devinsky O, Seshadri S and Friedman D: Incidence of seizures following initial ischemic stroke in a community-based cohort: The Framingham Heart Study. Seizure 47: 105-110, 2017.

2. Wang H, Wang B, Normoyle KP, Jackson K, Spitler K, Sharrock MF, Miller CM, Best C, Llano D and Du R: Brain temperature and its fundamental properties: A review for clinical neuroscientists. Front Neurosci 8: 307, 2014.

3. Lei Z, Zhang H, Liang Y and Xu ZC: Reduced expression of IA channels is associated with post-ischemic seizures. Epilepsy Res 124: 40-48, 2016.

4. Assis TM, Bacellar A, Costa G and Nascimento OJ: Mortality predictors of epilepsy and epileptic seizures among hospitalized elderly. Arq Neuropsiquiatr 73: 510-515, 2015.

5. Robertson CM and Perlman M: Follow-up of the term infant after hypoxic-ischemic encephalopathy. Paediatr Child Health 11: 278-282, 2006.

6. Glass HC, Glidden D, Jeremy RJ, Barkovich AJ, Ferriero DM and Miller SP: Clinical neonatal seizures are independently associated with outcome in infants at risk for hypoxic-ischemic brain injury. J Pediatr 155: 318-323, 2009. 
7. Procaccianti G, Zaniboni A, Rondelli F, Crisci M and Sacquegna T: Seizures in acute stroke: Incidence, risk factors and prognosis. Neuroepidemiology 39: 45-50, 2012.

8. Jacobs SE, Berg M, Hunt R, Tarnow-Mordi WO, Inder TE and Davis PG: Cooling for newborns with hypoxic ischaemic encephalopathy. Cochrane Database Syst Rev: CD003311, 2013.

9. Azzopardi DV, Strohm B, Edwards AD, Dyet L, Halliday HL, Juszczak E, Kapellou O, Levene M, Marlow N, Porter E, et al: Moderate hypothermia to treat perinatal asphyxial encephalopathy. N Engl J Med 361: 1349-1358, 2009.

10. Harbert MJ, Tam EW, Glass HC, Bonifacio SL, Haeusslein LA, Barkovich AJ, Jeremy RJ, Rogers EE, Glidden DV and Ferriero DM: Hypothermia is correlated with seizure absence in perinatal stroke. J Child Neurol 26: 1126-1130, 2011.

11. D'Ambrosio R, Eastman CL, Darvas F, Fender JS, Verley DR, Farin FM, Wilkerson HW, Temkin NR, Miller JW, Ojemann J, et al: Mild passive focal cooling prevents epileptic seizures after head injury in rats. Ann Neurol 73: 199-209, 2013.

12. Dietrich WD and Bramlett HM: The evidence for hypothermia as a neuroprotectant in traumatic brain injury. Neurotherapeutics 7 : 43-50, 2010.

13. Lasarge CL and Danzer SC: Mechanisms regulating neuronal excitability and seizure development following mTOR pathway hyperactivation. Front Mol Neurosci 7: 18, 2014.

14. Wong M: mTOR as a potential treatment target for epilepsy. Future Neurol 7: 537-545, 2012.

15. Butler CR, Boychuk JA and Smith BN: Effects of rapamycin treatment on neurogenesis and synaptic reorganization in the dentate gyrus after controlled cortical impact injury in mice. Front Syst Neurosci 9: 163, 2015.

16. Marin-Valencia I, Good LB, Ma Q, Duarte J, Bottiglieri T, Sinton CM, Heilig CW and Pascual JM: Glut1 deficiency (G1D): Epilepsy and metabolic dysfunction in a mouse model of the most common human phenotype. Neurobiol Dis 48: 92-101, 2012.

17. Pascual JM, Campistol J and Gil-Nagel A: Epilepsy in inherited metabolic disorders. Neurologist 14 (6 Suppl 1): S2-S14, 2008.

18. Ullner PM, Di Nardo A, Goldman JE, Schobel S, Yang H, Engelstad K, Wang D, Sahin M and De Vivo DC: Murine Glut-1 transporter haploinsufficiency: Postnatal deceleration of brain weight and reactive astrocytosis. Neurobiol Dis 36: 60-69, 2009.

19. Liemburg-Apers DC, Wagenaars JA, Smeitink JA, Willems PH and Koopman WJ: Acute stimulation of glucose influx upon mitoenergetic dysfunction requires LKB1, AMPK, Sirt2 and mTOR-RAPTOR. J Cell Sci 129: 4411-4423, 2016.

20. Demel HR, Feuerecker B, Piontek G, Seidl C, Blechert B, Pickhard A and Essler M: Effects of topoisomerase inhibitors that induce DNA damage response on glucose metabolism and $\mathrm{PI} 3 \mathrm{~K} / \mathrm{Akt} / \mathrm{mTOR}$ signaling in multiple myeloma cells. Am J Cancer Res 5: 1649-1664, 2015.

21. Inglis DJ, Lavranos TC, Beaumont DM, Leske AF, Brown CK, Hall AJ and Kremmidiotis G: The vascular disrupting agent BNC105 potentiates the efficacy of VEGF and mTOR inhibitors in renal and breast cancer. Cancer Biol Ther 15: 1552-1560, 2014.
22. Jiang X, Kenerson H, Aicher L, Miyaoka R, Eary J, Bissler J and Yeung RS: The tuberous sclerosis complex regulates trafficking of glucose transporters and glucose uptake. Am J Pathol 172: $1748-1756,2008$

23. Committee for the Update of the Guide for the Care and Use of Laboratory Animals, Institute for Laboratory Animal Research, National Research Council: Guide for the Care and Use of Laboratory Animals. 8th edition. The National Academies Press, Washington, DC, 2011.

24. Kawai K, Nitecka L, Ruetzler CA, Nagashima G, Joó F, Mies G, Nowak TS Jr, Saito N, Lohr JM and Klatzo I: Global cerebral ischemia associated with cardiac arrest in the rat: I. Dynamics of early neuronal changes. J Cereb Blood Flow Metab 12: 238-249, 1992.

25. Cao L, Tian Y, Jiang Y, Zhang GJ, Lei H and Di ZL: Down-regulation of Homerlb/c protects against chemically induced seizures through inhibition of mTOR signaling. Cell Physiol Biochem 35: 1633-1642, 2015.

26. Wu C, Wang J, Peng J, Patel N, Huang Y, Gao X, Aljarallah S, Eubanks JH, McDonald R and Zhang L: Modeling early-onset post-ischemic seizures in aging mice. Exp Neurol 271: 1-12, 2015.

27. Liu F, Benashski SE, Persky R, Xu Y, Li J and McCullough LD: Age-related changes in AMP-activated protein kinase after stroke. Age (Dordr) 34: 157-168, 2012.

28. Fu L, Huang L, Cao C, Yin Q and Liu J: Inhibition of AMP-activated protein kinase alleviates focal cerebral ischemia injury in mice: Interference with mTOR and autophagy. Brain Res 1650: 103-111, 2016.

29. Andresen M, Gazmuri JT, Marín A, Regueira T and Rovegno M: Therapeutic hypothermia for acute brain injuries. Scand J Trauma Resusc Emerg Med 23: 42, 2015.

30. Luo M, Li Q, Dong W, Zhai X and Kang L: Evaluation of mild hypothermia therapy for neonatal hypoxic-ischaemic encephalopathy on brain energy metabolism using $18 \mathrm{~F}$-fluorodeoxyglucose positron emission computed tomography. Exp Ther Med 8: 1219-1224, 2014

31. Bakhsheshi MF, Diop M, Morrison LB, St Lawrence K and Lee TY: Coupling of cerebral blood flow and oxygen consumption during hypothermia in newborn piglets as measured by time-resolved near-infrared spectroscopy: A pilot study. Neurophotonics 2: 035006, 2015.

32. Feng JZ, Wang WY, Zeng J, Zhou ZY, Peng J, Yang H, Deng PC, Li SJ, Lu CD and Jiang H: Optimization of brain metabolism using metabolic-targeted therapeutic hypothermia can reduce mortality from traumatic brain injury. J Trauma Acute Care Surg 83: 296-304, 2017.

This work is licensed under a Creative Commons Attribution-NonCommercial-NoDerivatives 4.0 International (CC BY-NC-ND 4.0) License. 